\title{
The POLAR Gamma-Ray Burst Polarimeter onboard the Chinese Spacelab
}

\author{
Silvio Orsi ${ }^{a}$, Franck Cadoux ${ }^{a}$, Catherine Leluc ${ }^{a}$, Mercedes Paniccia ${ }^{a}$, Martin Pohl ${ }^{a}$, Divic \\ Rapin $^{a}$, Neal Gauvin ${ }^{b}$, Nicolas Produit $^{b}$, Tianwei Bao ${ }^{c}$, Junying Chai $^{c}$, Yongwei Dong ${ }^{c}$, \\ Minnan Kong ${ }^{c}$, Lu Li ${ }^{c}$, Jiangtao Liu ${ }^{c}$, Xin Liu ${ }^{c}$, Haoli Shi ${ }^{c}$, Jianchao Sun ${ }^{c}$, Ruijie Wang ${ }^{c}$, Xing \\ $\mathrm{Wen}^{c}$, Bobing $\mathrm{Wu}^{b}$, Hualin Xiao ${ }^{c}$, Hanhui Xu ${ }^{c}$, Li Zhang ${ }^{c}$, Laiyu Zhang ${ }^{c}$, Shuangnan Zhang ${ }^{c}$, \\ Yongjie Zhang $^{c}$, Ilia Britvich ${ }^{d}$, Wojtek Hajdas ${ }^{d}$, Radoslaw Marcinkowski ${ }^{d}$, Dominik K. \\ Rybka $^{d, e}$ Tadeusz Batsch $^{e}$, Aleksandra Rutczynska ${ }^{e}$, Jacek Szabelski ${ }^{e}$ and Ania Zwolinska ${ }^{e}$, \\ ${ }^{a}$ DPNC, University of Geneva, quai Ernest-Ansermet 24, 1205 Geneva, Switzerland, and \\ Center for Astroparticle Physics (CAP), Geneva, Switzerland \\ ${ }^{b}$ ISDC, University of Geneva, Versoix, Switzerland, and Center for Astroparticle Physics \\ (CAP), Geneva, Switzerland \\ ${ }^{c}$ Institute of High Energy Physics, Beijing, China \\ ${ }^{d}$ PSI, Villigen, Switzerland \\ e National Center for Nuclear Research (NCBJ), Poland
}

\begin{abstract}
POLAR is a joint European-Chinese experiment aimed at a precise measurement of hard X-ray polarization $(50-500 \mathrm{keV})$ of the prompt emission of Gamma-Ray Bursts. The main aim is a better understanding of the geometry of astrophysical sources and of the X-ray emission mechanisms. POLAR is a compact Compton polarimeter characterized by a large modulation factor, effective area, and field of view. It consists of 1600 low-Z plastic scintillator bars read out by 25 flat-panel multi-anode photomultipliers. The incoming X-rays undergo Compton scattering in the bars and produce a modulation pattern; experiments with polarized synchrotron radiation and GEANT4 Monte Carlo simulations have shown that the polarization degree and angle can be retrieved from this pattern with the accuracy necessary for identifying the GRB mechanism. The flight model of POLAR is currently under construction in Geneva. The POLAR instrument will be placed onboard the Chinese spacelab TG-2, scheduled for launch in low Earth orbit in 2015. The main milestones of the space qualification campaign will be described in the paper.
\end{abstract}

Keywords: X-ray polarization, Scintillator detector, Synchrotron radiation, Astrophysics, Gamma-Ray Burst (GRB), GEANT4 Monte Carlo simulations, Compton polarimeter, Space experiment

\section{INTRODUCTION}

Gamma Ray Bursts are considered the most energetic events in our Universe after the Big Bang. They are sudden flashes of $\gamma$-rays that appear randomly in the sky from distant galaxies and outshine for a few seconds all other $\gamma$-ray sources. In the past years many instruments have performed extensive studies of GRBs, but their emission mechanism and their progenitors are still debated. The direction and the level of polarization of highenergy photons emitted by GRBs are very good observable candidates for the understanding of the corresponding creation mechanisms, geometry of the GRB core and strength of magnetic fields at work. ${ }^{1}$

$\mathrm{POLAR}^{2}$ is a space-borne experiment, built within a European-Chinese collaboration, aimed at a precise measurement of hard X-ray polarization of the prompt emission of GRBs. Thanks to its large modulation factor, wide field of view (1/3 of the visible sky), and large effective area, POLAR will be able to reach a minimum detectable polarization (1- $\sigma$ level) of about $3 \%$ for several GRB measurements per year.

Further author information: (Send correspondence to Silvio Orsi)

Silvio Orsi: E-mail: Silvio.Orsi@cern.ch, Telephone: +41 223796923

Space Telescopes and Instrumentation 2014: Ultraviolet to Gamma Ray, edited by Tadayuki Takahashi, Jan-Willem A. den Herder, Mark Bautz, Proc. of SPIE Vol. 9144, 91440M · C 2014 SPIE CCC code: $0277-786 \mathrm{X} / 14 / \$ 18 \cdot$ doi: $10.1117 / 12.2055910$

Proc. of SPIE Vol. $914491440 \mathrm{M}-1$ 


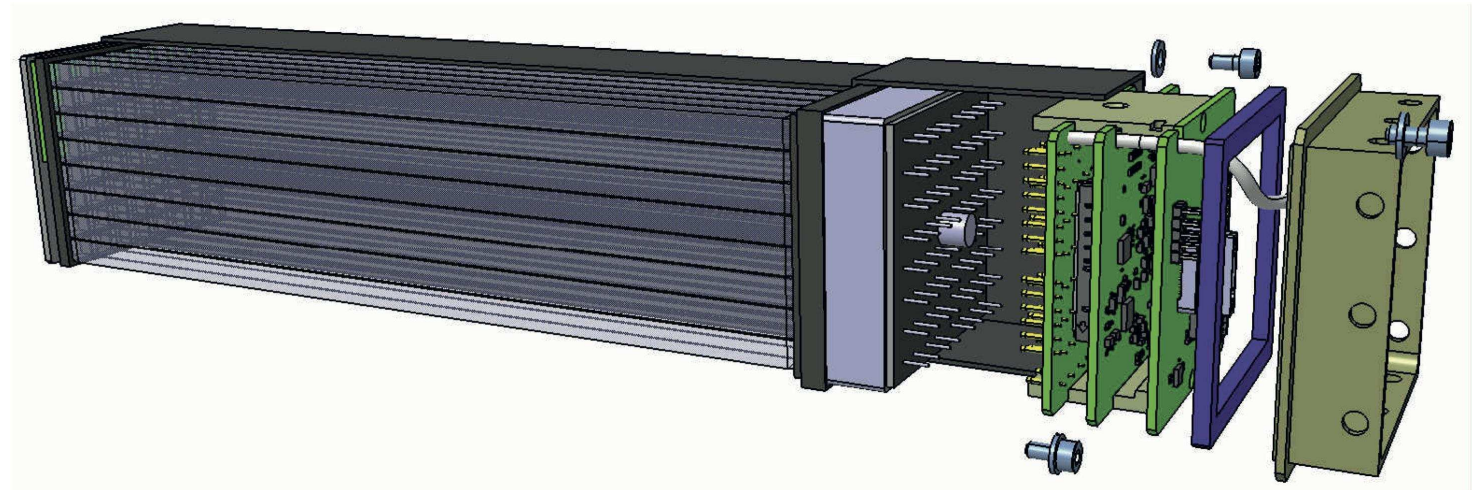

Figure 1. A modular unit of the POLAR detector. From left to right, the figure shows: scintillator bars, optical pad, MAPMT, FEE (green), thermal seal (blue). A carbon fiber socket (black) surrounds the unit and an aluminum 'end cap' (on the right) provides mechanical fixation.

\section{THE POLAR DETECTOR}

POLAR is a novel polarimeter consisting of a target of $40 \times 40$ plastic scintillator bars (Eljen EJ248M), each of dimension $5.9 \times 5.9 \times 176 \mathrm{~mm}^{3}$, wrapped in a highly reflective foil (Vikuiti ESR, 3M), organized in 25 independent modular units, with 64 bars each. The ends of the bars are shaped as a truncated cone, in order to reduce optical crosstalk between the channels. In each modular unit, the energy deposited in the bars is converted into optical photons, which travel through the scintillator bars and are collected into flat panel multi anode photomultiplier tubes (MAPMT; H8500, Hamamatsu), mechanically coupled to the bottom of the scintillator bars via a thin transparent optical pad, and enclosed in a $1 \mathrm{~mm}$ carbon fiber socket. The electrical signals from the MAPMT are first processed by an ASIC and FPGA at the front-end electronics (FEE), then sent to a central processing unit, where the trigger decision is taken based on the outputs of all modular units. Data are then stored onboard, before being downlinked to Earth for analysis. The expected data flow from POLAR to ground is about 5 GB per day. This modular design provides a good mechanical stability and facilitates the interchange of modules during the testing phase of the detector. The bars in each modular unit are kept together with two black aligning plastic frames located at the top and at the bottom of the carbon fiber sockets to provide resistance to vibrations and to reduce the optical crosstalk between adjacent channels. Figure 1 shows an exploded view of a modular unit. The scintillator bars are enclosed in a $3 \mathrm{~mm}$ thick carbon fiber box that enhances the mechanical stability and acts as a shield against low energy charged cosmic rays, while the electronics subsystems (central computer and low/high voltage power supplies) are inserted in an aluminum frame. POLAR has a resistant and redundant design that allows it to withstand the harsh environment that the apparatus will face: it has to survive to strong vibrations during launch, and pyro-shocks when the stages detach from the rocket and when solar panels open. POLAR has also to mitigate the effects of the heat from the Sun in low Earth orbit, which may lead to temperature variations of $200^{\circ} \mathrm{C}$ for unprotected devices that face deep space.

Figure 2 provides a $\mathrm{CAO}$ view of the detector. The detector is located on the outside of the spacecraft and faces deep space, as shown in figure 3. Since the Earth atmosphere is opaque to X-rays coming from space, photons in the energy range between 50 and $500 \mathrm{keV}$ originating from GRBs cannot reach the Earth surface; it was therefore decided that POLAR would be a space-born experiment. POLAR is scheduled for launch onboard the future Chinese Tian-Gong 2 Spacelab in 2015.

\section{GAMMA-RAY INTERACTION IN POLAR}

X-rays from a GRBs enter the active volume of POLAR and undergo Compton scattering in one of the 1600 bars, while the scattered photon interacts via Compton or photoelectric effect in a second bar, as schematically shown in figure 4. Photons tend to Compton scatter at right angles to the incident polarization vector, according 


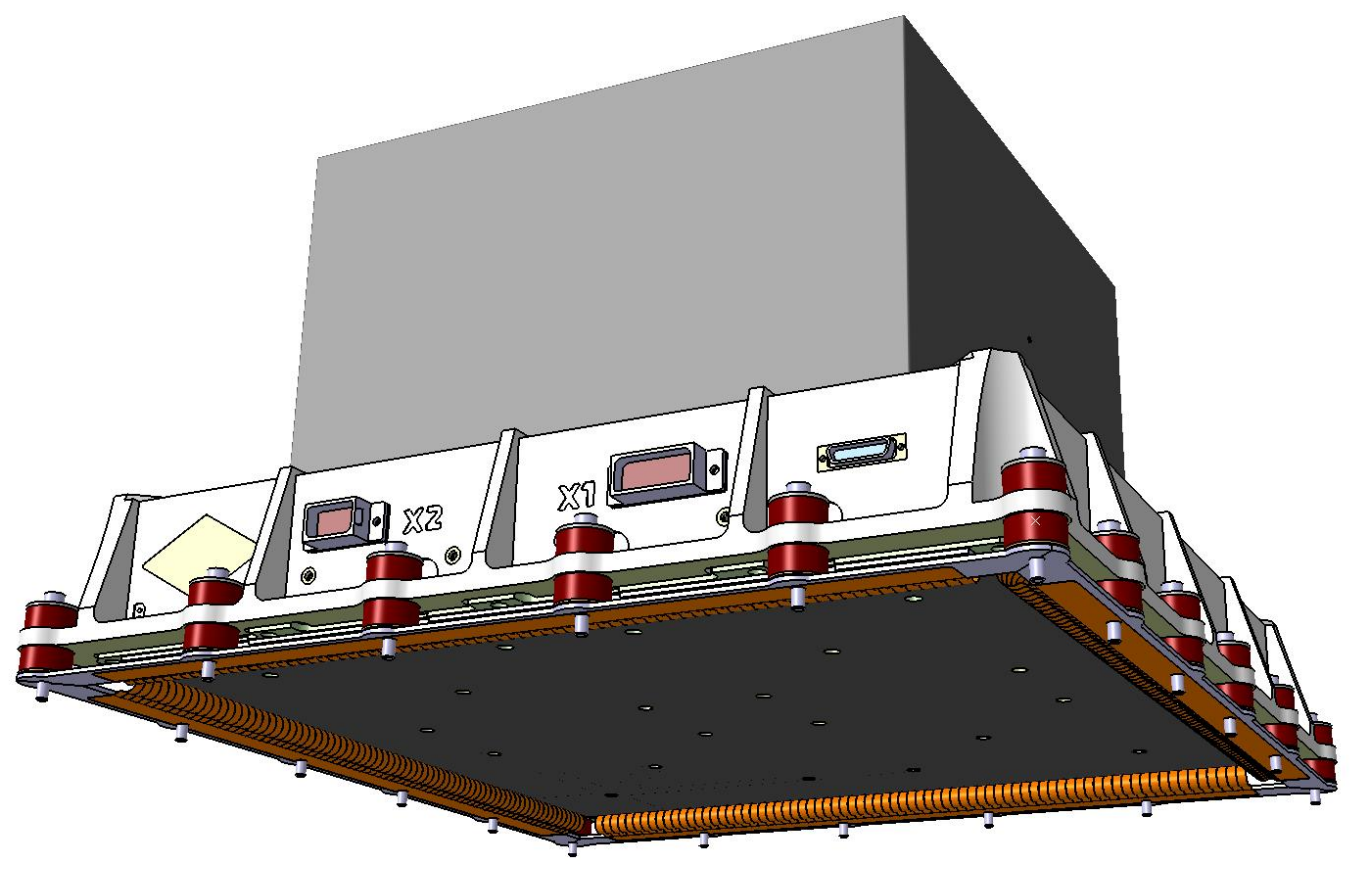

Figure 2. The POLAR detector.

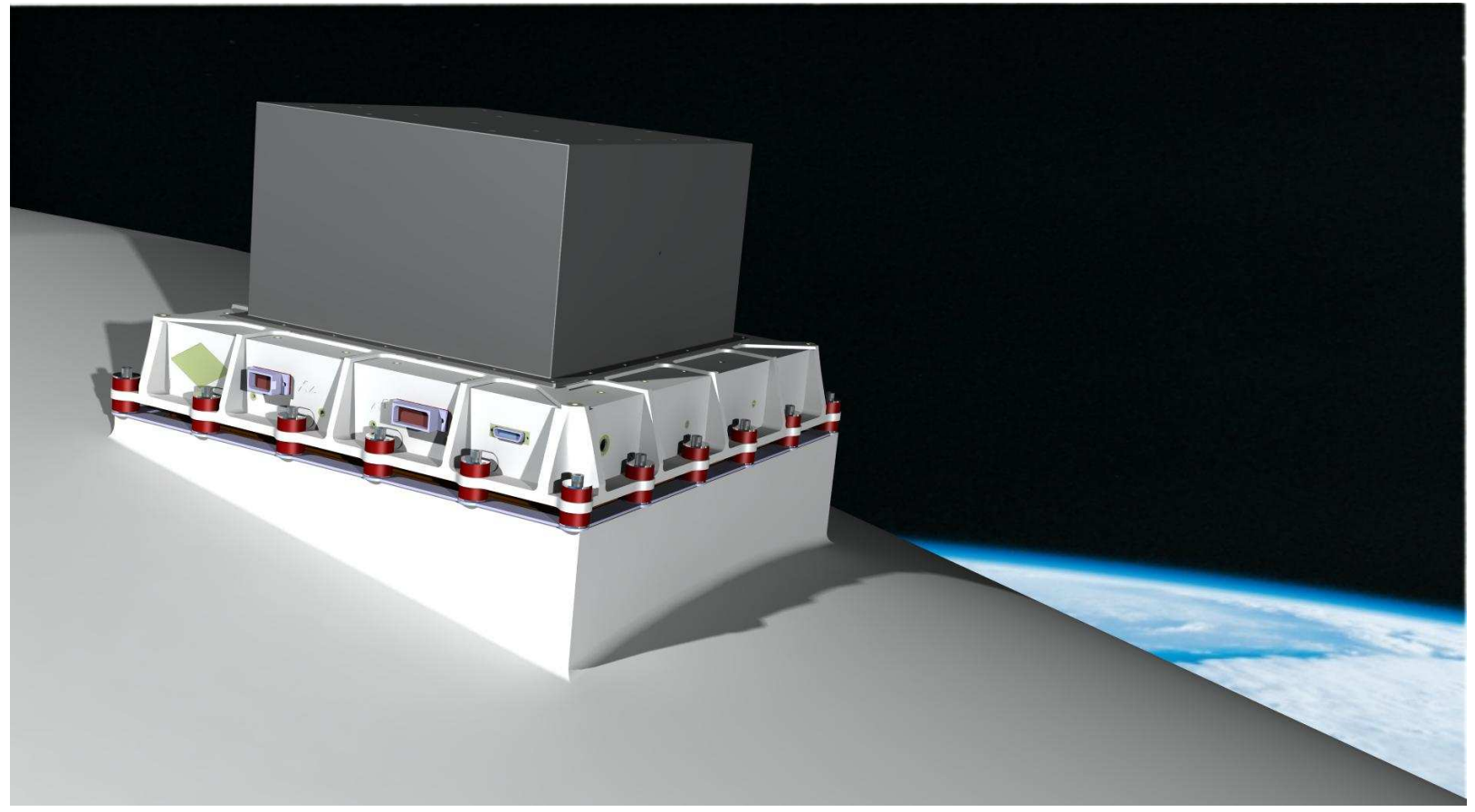

Figure 3. Artistic view of POLAR installed on the Chinese spacelab Tian-Gong 2.

Proc. of SPIE Vol. $914491440 \mathrm{M}-3$

Downloaded From: https://www.spiedigitallibrary.org/conference-proceedings-of-spie on 19 Apr 2019 Terms of Use: https://www.spiedigitallibrary.org/terms-of-use 

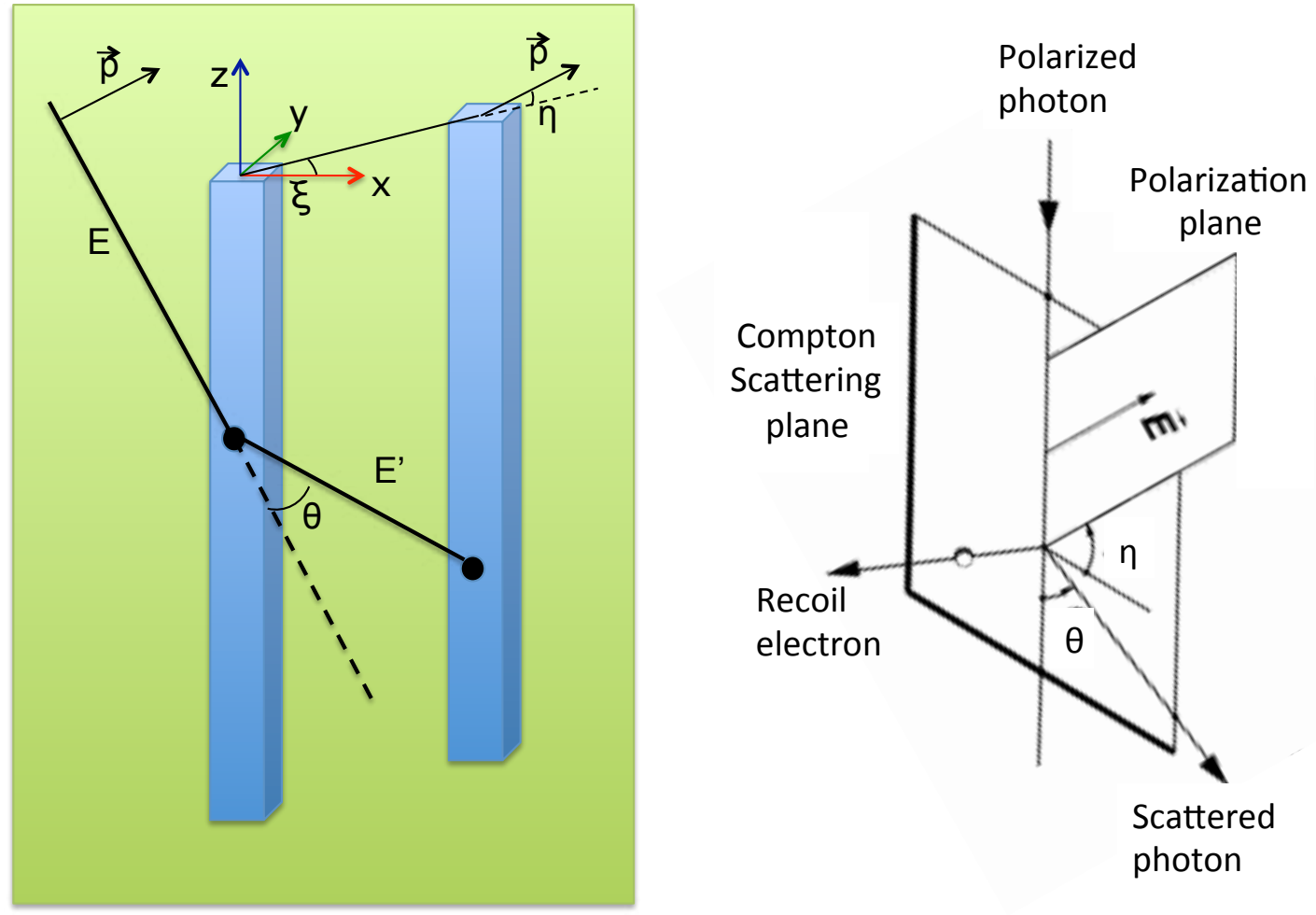

Figure 4. (Left) Particle interaction in POLAR. The incoming X-ray interacts via Compton scattering in a bar, and the scattered photon interacts (via Compton or photoelectric effect) in a different bar. (Right) Kinematics of Compton scattering. The vectors and planes relative to the principal quantities involved in the scattering process are shown.

to the Klein-Nishina equation:

$$
\frac{d \sigma}{d \Omega}(\theta, \eta)=\frac{r_{0}^{2}}{2}\left(\frac{E^{\prime}}{E}\right)^{2}\left(\frac{E}{E^{\prime}}+\frac{E^{\prime}}{E}-2 \sin ^{2} \theta \cos ^{2} \eta\right)
$$

where $E$ and $E^{\prime}$ are respectively the initial and final photon energies and $r_{0}$ is the classic electron radius; the Compton scattering angle $\theta$ and the Azimuthal angle $\eta$ are defined in figure 4 (left). We define the angle $\xi$ (see figure 4) as the azimuthal scattering angle measured with respect to the $x$-axis of the laboratory reference system. The distribution of the angle $\xi$ measured on a large sample of data has a sinusoidal behavior with period $\pi$ and provides the expectation value $\eta$, i.e. the information on the polarization of the photon sample:

$$
f(\xi)=K \cdot\left\{1+\mu \cos \left[2\left(\xi-\xi_{0}\right)+\pi\right]\right\},
$$

where $K$ is a normalization constant, $\mu$ is the modulation factor, $\xi_{0}$ is the polarization angle and the term $\pi$ is added in order to make the angle $\xi_{0}$ assume the expectation value $\eta$. The angle of polarization $\left(\xi_{0}\right)$ and the modulation factor $(\mu)$ are obtained from a fit to the data, while the degree of linear polarization is $\Pi=\mu / \mu_{100}$, where $\mu_{100}$ is the modulation factor for a $100 \%$ polarized photon beam, obtained with Monte Carlo simulations and verified with beam test data. Data from a polarized photon beam contain therefore a modulation pattern; experiments with polarized synchrotron radiation and GEANT4 Monte Carlo simulations have shown that the polarization degree and angle can be retrieved from this pattern with the accuracy necessary for pinning down the GRB mechanisms.

\section{CALIBRATION WITH SYNCHROTRON RADIATION}

Several experiments with polarized synchrotron radiation were performed in the past years at the high energy diffraction and scattering beam line ID15A at the European Synchrotron Radiation Facility (ESRF), in Grenoble 
(France), which provides $100 \%$ polarized X-rays in the energy range between $50 \mathrm{keV}$ and $\sim 700 \mathrm{keV}$, creating ideal conditions for the calibration of a hard X-ray polarimeter as POLAR. Other experiments took place at the Swiss Light Source (SLS) at the Paul Scherrer Institute in Villigen (Switzerland) and at the Shanghai Synchrotron Radiation Facility (SSRF) in Shanghai (China). The typical experiment consists of recording all pairs of bars that show a coincident energy deposition. From the distribution of the azimuthal scattering angle $\xi$ we have reconstructed the angle of polarization $\xi_{0}$ of the incoming photon beam and the modulation factor $\mu_{100}$, i.e. the response of the instrument to a fully polarized photon flux. During each experiment data were collected with the beam directed sequentially into each bar, which allows to approximately reproduce the flat illumination expected from a GRB. Several rotations around the beam axis allowed to test the response of the detector to several polarization angles.

The first experiment was devoted to assess the performance of one modular unit of POLAR with high-energy synchrotron radiation (ESRF, 2009). ${ }^{3}$ In order to detect photons with incoming energies around $50 \mathrm{keV}$ via Compton scattering, energy depositions as low as $5 \mathrm{keV}$ per channel are recorded in the apparatus. For this reason one experiment was devoted to investigating the detection of photons near the detection threshold with low-energy X-rays (SLS, 2011). Following experiments (ESRF, 2011-2014) were aimed at studying the interaction between modular units with polarized X-rays over a wide energy range. One calibration of several modular units with $70 \mathrm{keV}$ photons was done in 2014 in SSRF (China). The last experiment (ESRF, April 2014) was performed to calibrate in detail one modular unit of the POLAR flight model over a wide energy range ( $80-535 \mathrm{keV})$. Data analysis is currently ongoing. The high quality of the data collected during the above mentioned experiments confirmed that the chosen geometry allows POLAR to reach its scientific goals, as predicted by Monte Carlo simulations. These results confirm also the good performance of the read-out electronics.

\section{POLAR AND THE SPACE ENVIRONMENT}

The space environment is very harsh: all chosen materials must be resistant to ionizing radiation due to the presence of cosmic rays; all external surfaces must have a special treatment because the heat coming from the Sun (1 solar constant, i.e. $\sim 1400 \mathrm{~W} / \mathrm{m}^{2}$ in low Earth orbit) and because deep space behaves like a black body with extremely low temperature $(\sim 3 \mathrm{~K})$. The external surfaces of POLAR made of aluminum are painted with a space-qualified white paint (MAP, France) with high reflectivity (0.8) for solar light and low emissivity $(0.2)$ in the infrared region. A sheet of multi layer insulator (MLI) covers completely the surfaces in carbon fiber, to reduce to the minimum any heat exchange through the top part of the apparatus. Temperature variations between different orbits (due to large variations in Sun exposure, and due to the orbit parameters - namely the $\beta$ angle) make necessary to test the detector in the most extreme temperature conditions. Launch and dockings in orbit induce vibrations and shocks, which imposes to perform vibration tests on a hydrodynamic shaker, and shock tests with a dedicated pyro-shock facility. Twenty shock attenuators were inserted in the design of POLAR, in order to reduce shock waves propagating from the rocket (during launch) and from the spacecraft (during dockings) to a level acceptable for the most delicate components, i.e. the photomultipliers. Several pyro-shock tests showed that these attenuators perform according to their expectations.

\section{SPACE QUALIFICATION: ENVIRONMENTAL TESTS}

All parts of POLAR were tested to the conditions expected in space. In particular, all electronic components were selected to be radiation resistant; they were either tested and used by previous experiments or guaranteed by the producer. One FEE unit was irradiated at the HUG hospital in Geneva with $6 \mathrm{MeV}$ photons for a total dose of $1 \mathrm{krad}$, while critical components of the low voltage power supply were tested with the same photon beam up to a total dose of $9 \mathrm{krad}$. A complete space qualification campaign was performed on a full-scale qualification model of POLAR, and is detailed in the subsections below.

\subsection{Thermal Design and Tests}

The thermal design of POLAR involves passive cooling, where heat is transferred between POLAR, the spacecraft and deep space. At the interface between POLAR and the spacecraft, straps of highly thermally conductive materials are present: copper and pyrolytic graphite sheet (PGS, by Panasonic). These straps ensure that heat generated inside POLAR $(\sim 45 \mathrm{~W})$ during physics operation is removed from the detector, and that heat from 


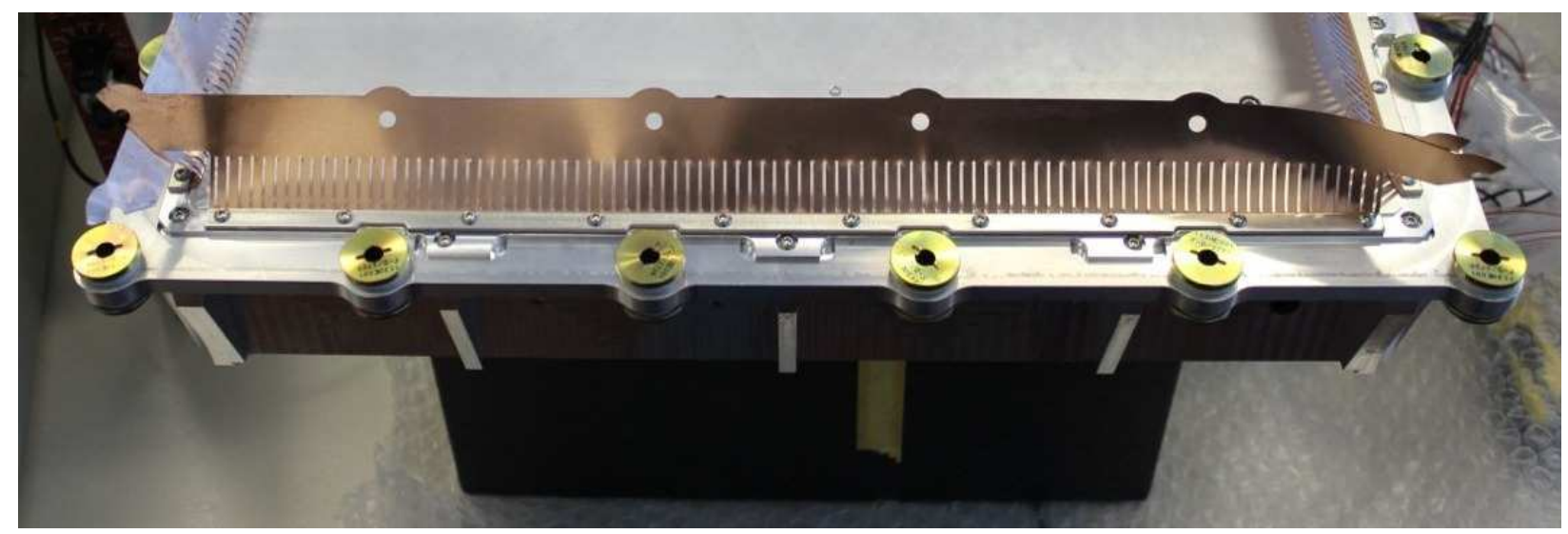

Figure 5. Detail of the copper straps present between POLAR and the spacelab Tian-Gong 2.

the spacecraft flows into the detector during cold periods, such as when POLAR is switched off during docking of spacecrafts to the TG-2 spacelab. When POLAR is off, the temperature inside POLAR may drop too much. Four heaters generating a total power of $20 \mathrm{~W}$ are therefore inserted in the design to prevent damages in the electronics due to excessively low temperatures. Heaters turn on automatically when the temperature inside POLAR drops below $-30^{\circ} \mathrm{C}$. One of the copper straps is visible in the photo in figure 5 ; the four straps are shown as orange lines at the sides of the detector in figure 2 (CAO image). The white paint on the outer sides of POLAR ensures also that the solar rays are mostly reflected (low absorption coefficient), and that heat from POLAR is emitted towards deep space (high emissivity).

To verify the thermal design of POLAR, several tests were performed first at the subsystem level, and then at the system level. The main tests done to ensure that POLAR will operate and perform correctly in orbit and also to validate the thermal simulations are:

- Thermal test: The qualification model of POLAR was placed in a thermal chamber; the temperature of the chamber varied between low and high limits $\left(26.5\right.$ cycles between $-35^{\circ} \mathrm{C}$ to $+45^{\circ} \mathrm{C}$, with a period of approximately 12 hours. The test verified the correct functioning of the detector over the whole range of temperatures to which POLAR will be exposed in space and simulated an accelerated aging of the various parts. The correct operation of the detector is continuously monitored during the test, and functional tests are performed at every cycle.

- Thermal vacuum test: The detector was placed inside a vacuum chamber (at a pressure of few times $10^{-5}$ mbar). The experimental setup is shown in figure 6. Heat is transferred between POLAR and the chamber through the cold plate (via an aluminum interface plate, which represents the Tian-Gong 2 interface) and via the shroud, whose temperatures could be set separately. In practice, we always set both cold plate and shroud to identical temperature. Several cycles were performed, where each cycle takes about 24 hours.

- A thermal balance test is planned for the summer 2014 in a thermal vacuum chamber equipped with a solar simulator (1 solar constant) to verify experimentally the thermal simulations in conditions very similar to the ones that POLAR will encounter in orbit.

\subsection{Electromagnetic Compatibility Test}

Electromagnetic compatibility tests have been successfully performed with the Laboratory of Electromagnetic Characterization of the "Polo Scientifico e Didattico di Terni", University of Perugia, Faculty of Engineering in Terni, Italy. The test procedure for each test is in accordance with requirements from Chinese authorities and with reference document MIL-STD 461E. The purpose of the test is to verify that 1) the electromagnetic emission is within given limits (i.e. POLAR does not damage or perturb the operation of other instruments onboard the 


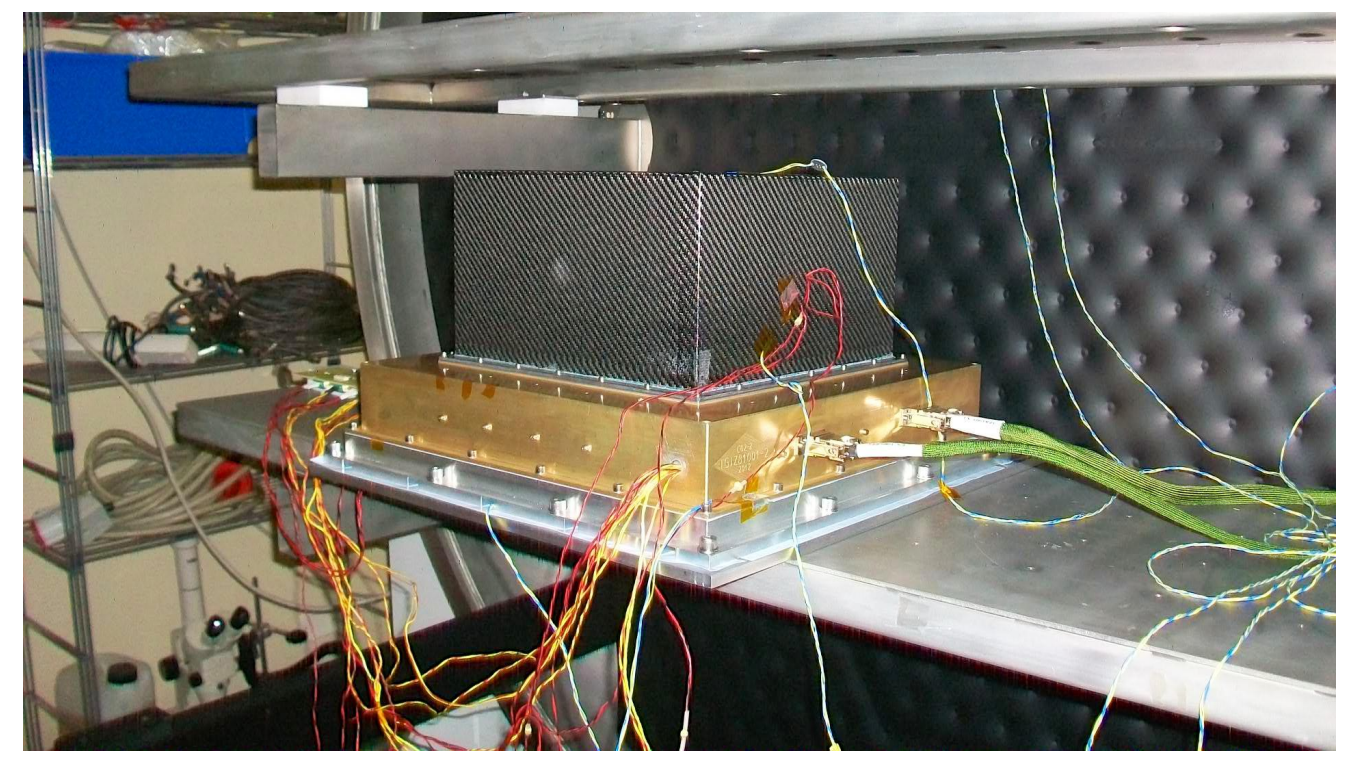

Figure 6. Setup of the thermal vacuum test of the qualification model of POLAR that took place in May 2014. POLAR is installed on the cold plate. Data and power are transferred to/from POLAR via two connectors (the cables are visible in green in the online photo).

spacelab), and that 2) POLAR is not affected by incoming electromagnetic radiation (i.e. POLAR operation is not perturbed by other nearby instruments). The list of performed tests is shown in table 1 .

\subsection{Mechanical Tests}

The following mechanical tests were performed at the system level, as part of the qualification campaign of POLAR, as required by Chinese authorities:

- A static acceleration test was performed in 2013, with an acceleration of $6.5 \mathrm{~g}$ over the three axes, in a large circular centrifuge. This test emulates the constant acceleration during ascent of the rocket.

- Sine sweep: The test is performed between $4 \mathrm{~Hz}$ and $100 \mathrm{~Hz}$ over the 3 axes on a hydrodynamic shaker (with a maximum amplitude of $9.8 \mathrm{~g}$ in the frequency range $17-60 \mathrm{~Hz}$. This test is intended to verify that low-frequency resonances in the detector do not damage the device (or even the rocket due to parts that may detach from the system) during launch. It should be noted that the shock attenuators have low eigenfrequencies (between 60 and $90 \mathrm{~Hz}$ ), in order to increase the damping effect above $400 \mathrm{~Hz}$, as required to protect the photomultiplier windows.

Table 1. List of EMC tests performed during the qualification campaign of POLAR.

\begin{tabular}{|l|}
\hline Test \\
\hline CE 102 Conducted emission \\
\hline CS 101 Conducted susceptibility-audio frequency \\
\hline CS 114 Conducted susceptibility-radio frequency \\
\hline CS 115 Conducted susceptibility-pulses \\
\hline CS 116 Conducted susceptibility-damped sinusoidal transients \\
\hline RE 102 Radiated emission-electric field \\
\hline RS 103 Radiated susceptibility-electric field \\
\hline
\end{tabular}




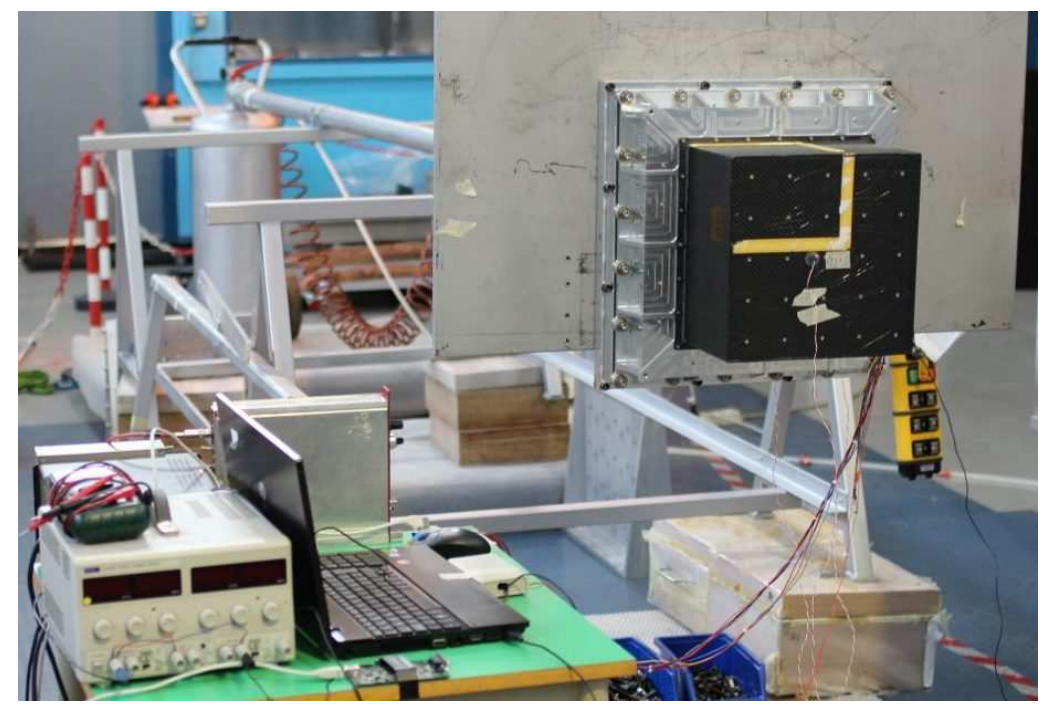

Figure 7. Pyro-shock test of POLAR. The shock is performed here along the longitudinal direction of the bars. A steel projectile is accelerated in the pipe visible on the top left, and hits the plate where POLAR is installed. The impact creates a shock with the desired shock response spectrum (SRS; see figure 8).

- Random vibrations with frequencies up to $2 \mathrm{kHz}$ were tested for 2 minutes for each axis, to reproduce the vibrations that POLAR will need to sustain during launch. Several tests, at the modular unit level and at the system level, were performed.

- Pyro-shock tests with intensity up to $500 \mathrm{~g}$ were performed at the modular unit level and at the system level in the S.E.R.M.S. laboratories in Terni, Italy. The setup is shown in figure 7. The insertion of 20 shock attenuators, designed after a first failure of the photomultipliers during the first pyro-shock test, effectively reduced the input shock levels to levels acceptable for all components in POLAR, as shown in figure 8. Other shock tests with hydrodynamic shakers were also performed in China and in France, to compare the two technologies.

Dedicated mechanical tests (sine sweep, random and pyro-shock) were performed on a reduced mass model of POLAR with 4 shock attenuators, in order to characterize the attenuators (figure 9). The response in frequency allowed us to choose between two types of attenuators. Several sine sweep runs at low amplitude (0.5 g) were realized before and after the above mentioned tests to search for any resonance change in the system due to the mechanical tests. Functional and performance tests were also performed before and after each mechanical test. The device passed successfully all the above mentioned mechanical tests.

\section{CONCLUSIONS AND OUTLOOK}

POLAR is a compact Compton polarimeter with a wide field of view dedicated to the study of the polarization of the prompt emission of Gamma Ray Bursts. The space qualification campaign ended successfully in May 2014, together with a series of calibration tests with synchrotron radiation, mostly at the European Synchrotron Radiation Facility (ESRF) in Grenoble, France. The flight model of the instrument is currently under construction, in view of a launch in 2015 onboard the Chinese spacelab Tian-Gong 2. The expected lifetime of POLAR is of three years .

\section{ACKNOWLEDGMENTS}

The development of the POLAR project is supported by the China Manned Space Agency (CMSA) and by the European Space Agency (ESA) through its PRODEX office. The authors acknowledge also the support of the European Synchrotron Radiation Facility (ESRF, Grenoble, France) and the Swiss Light Source (PSI, Villigen, 


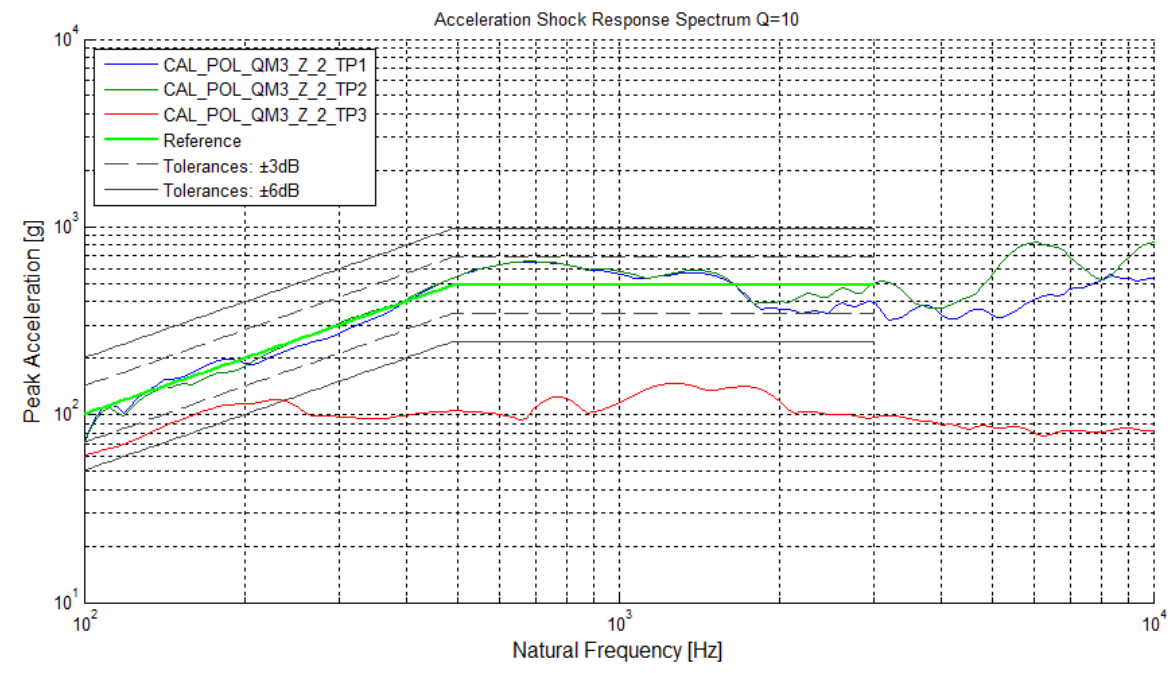

Figure 8. Shock response spectrum (SRS) of a pyro-shock test of POLAR. The reference line (green) indicates the required level (tolerance $6 \mathrm{~dB}$ ); the measured input, i.e. the measurements on the plate near the impact point (shown in dark green and blue) are well within the tolerances. The red line is a measurement of the acceleration on POLAR (output); the difference between output and input is due to the damping effect of the shock attenuators.

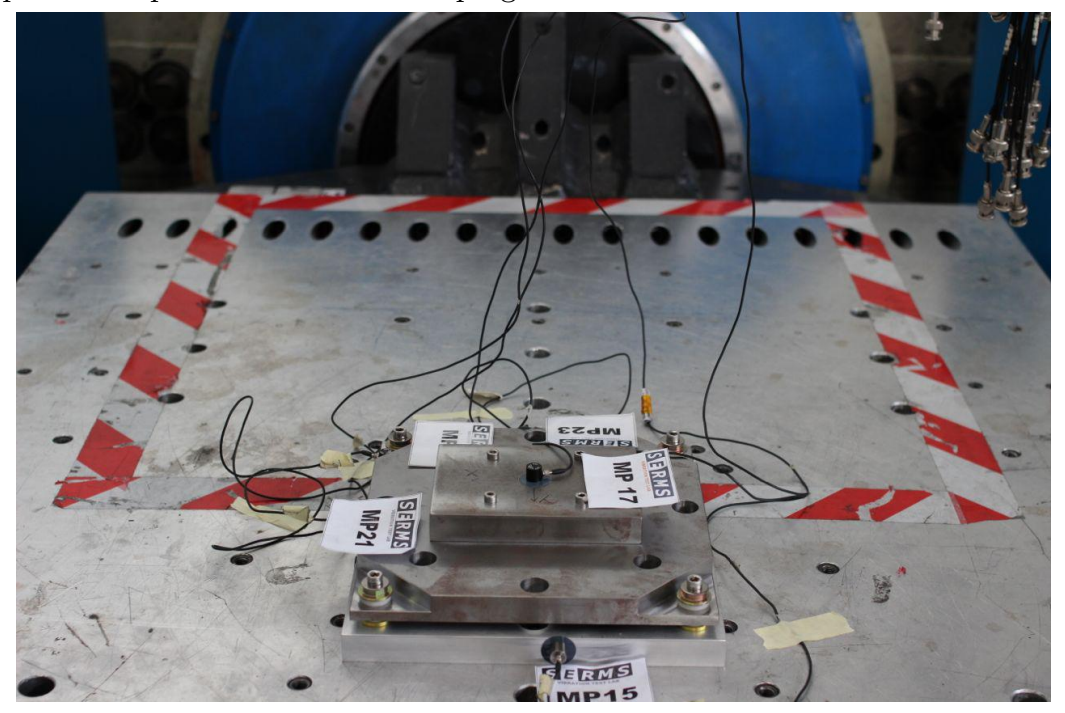

Figure 9. Vibration test on a shaker of one small mechanical model of POLAR; four shock attenuators were installed between the device and the interface plate. Several accelerometers register the accelerations along the 3 main axes.

Switzerland) for provision of synchrotron radiation facilities, and the financial support of of the Swiss National Science Foundation.

\section{REFERENCES}

[1] Toma K. et al., "Statistical Properties of Gamma-Ray Burst Polarization", Astrophys. J. 698, 1042-1053 (2009).

[2] Produit N. et al., "POLAR, a compact detector for gamma-ray bursts photon polarization measurements", NIM A 550, 616-625 (2005).

[3] Orsi S. et al., "Response of the Compton polarimeter POLAR to polarized hard X-rays", NIM A 648, 139-154 (2011). 